\title{
Quantification of Magnetic Surface and Edge States in an FeGe Nanostripe by Off-Axis Electron Holography
}

\author{
Dongsheng Song, ${ }^{1,3}$ Zi-An Li, ${ }^{2,3,}{ }^{*}$ Jan Caron, ${ }^{3}$ András Kovács, ${ }^{3}$ Huanfang Tian, ${ }^{2}$ Chiming Jin, ${ }^{4}$ \\ Haifeng Du, ${ }^{4}$ Mingliang Tian, ${ }^{4}$ Jianqi Li, ${ }^{2}$ Jing Zhu, ${ }^{1}$ and Rafal E. Dunin-Borkowski ${ }^{3}$ \\ ${ }^{1}$ National Center for Electron Microscopy in Beijing, Key Laboratory of Advanced Materials (MOE) and The State Key Laboratory of \\ New Ceramics and Fine Processing, School of Materials Science and Engineering, Tsinghua University, 100084 Beijing, China \\ ${ }^{2}$ Beijing National Laboratory for Condensed Matter Physics, Institute of Physics, Chinese Academy of Sciences, 100190 Beijing, China \\ ${ }^{3}$ Ernst Ruska-Centre for Microscopy and Spectroscopy with Electrons and Peter Grünberg Institute, \\ Forschungszentrum Jülich, 52425 Jülich, Germany \\ ${ }^{4}$ High Magnetic Field Laboratory, Chinese Academy of Sciences, 230031 Anhui, China
}

(Received 15 August 2017; revised manuscript received 13 December 2017; published 19 April 2018)

\begin{abstract}
Whereas theoretical investigations have revealed the significant influence of magnetic surface and edge states on Skyrmonic spin texture in chiral magnets, experimental studies of such chiral states remain elusive. Here, we study chiral edge states in an FeGe nanostripe experimentally using off-axis electron holography. Our results reveal the magnetic-field-driven formation of chiral edge states and their penetration lengths at 95 and $240 \mathrm{~K}$. We determine values of saturation magnetization $M_{S}$ by analyzing the projected in-plane magnetization distributions of helices and Skyrmions. Values of $M_{S}$ inferred for Skyrmions are lower by a few percent than those for helices. We attribute this difference to the presence of chiral surface states, which are predicted theoretically in a three-dimensional Skyrmion model. Our experiments provide direct quantitative measurements of magnetic chiral boundary states and highlight the applicability of state-ofthe-art electron holography for the study of complex spin textures in nanostructures.
\end{abstract}

DOI: 10.1103/PhysRevLett.120.167204

Introduction.-In certain physical systems, peculiar boundary states can arise because of topology and geometry considerations. Such boundary states include edge current modes in quantum Hall systems [1], edge metallic states in topological insulators [2], and spin boundary states in chiral magnets [3]. In chiral magnets, noncollinear spin textures at surfaces and edges surround magnetic Skyrmions: particlelike swirling spin textures [4]. Rohart and Thiaville have theorized [3] that the Dzyaloshinskii-Moriya interaction (DMI) $[5,6]$ in a chiral magnet can lead to specific micromagnetic boundary conditions that twist the magnetization at sample edges, with a feature length that is a fraction of a helix period [7]. Such edge spin configurations preserve the magnetic chirality around the sample edges and play a key role in current-induced Skyrmion motion in nanostripes [8]. Moreover, the enhanced stability of Skyrmions in systems that have reduced dimensions has been attributed theoretically to the presence of peculiar spin textures at chiral boundaries (surfaces and edges) [9,10]. Experimentally, chiral edge states have been inferred indirectly from magnetotransport measurements [11] and using magnetic imaging

Published by the American Physical Society under the terms of the Creative Commons Attribution 4.0 International license. Further distribution of this work must maintain attribution to the author(s) and the published article's title, journal citation, and DOI.
[12]. Given the importance of chiral boundary states for understanding Skyrmion physics and exploiting Skyrmionbased applications, it is important to experimentally quantify the local magnetic properties of chiral boundary states and their influence on Skyrmions in nanostructures.

Advanced magnetic imaging techniques that have been used to image magnetic Skyrmions include spin-polarized scanning tunneling microscopy (SP-STM) [13], x-ray magnetic circular dichroism in scanning transmission $\mathrm{x}$-ray microscopy (STXM) [14], and magnetic force microscopy (MFM) [15]. However, STXM and MFM are not well suited to resolving fine chiral edge and surface states because of their relatively poor spatial resolution (a few tens of nm). SPSTM has stringent requirements for clean material surfaces and probes only surface spins. Transmission electron microscopy (TEM) based techniques such as Lorentz microscopy (LM) [12,16], off-axis electron holography (EH) [17-19], and differential phase contrast (DPC) imaging [20,21] have been used to image magnetic Skyrmions in thin films with sub-10-nm spatial resolution. However, resolving Skyrmionic spin textures in nanostructures is highly challenging because of the required spatial resolution and magnetic sensitivity. In particular, the LM technique is not suitable for studying magnetic nanostructures that have sizes far below $100 \mathrm{~nm}$ because the formation of Fresnel fringes arising from changes in specimen thickness at the boundaries of nanostructures complicates interpretation of the magnetic 
contribution. Off-axis EH provides the phase of the electron wave function that has passed through a sample, allowing the magnetic signal to be separated more easily than using other TEM-based techniques when studying Skyrmions in confined nanostructures [22,23].

In this Letter, we use state-of-the-art off-axis EH to investigate the formation of magnetic edge states in an FeGe nanostripe in the presence of magnetic fields and as a function of temperature. We determine the projected inplane magnetization distributions of chiral edge twists and Skyrmions from recorded phase images using a newly developed magnetization reconstruction technique. We perform quantitative measurements from the reconstructed magnetization distributions, in order to assess the characteristic penetration lengths of edge twists, the sizes of confined Skyrmions, and their saturation magnetizations at different temperatures.

Experimental details. - A thin nanostripe of noncentrosymmetric single crystalline B20 FeGe was prepared using focused ion beam milling, as described elsewhere [12,22]. Structural characterization was performed using an FEI F20 TEM operated at $200 \mathrm{kV}$ and is given in the Supplemental Material [24] (Figs. S1 and S2). For magnetic imaging, we used an image-aberration-corrected FEI Titan 80-300 TEM equipped with a Lorentz lens and two electron biprisms. The use of a liquid-nitrogen-cooled specimen holder (Gatan model 636) allowed the sample temperature to be varied between 95 and $370 \mathrm{~K}$. The objective lens of the microscope was used to apply out-of-plane magnetic fields of between 0 and $1.5 \mathrm{~T}$. A cumulative acquisition approach was used to record 20 electron holograms, with an exposure time of $6 \mathrm{~s}$ for each hologram. The holograms were reconstructed by a standard Fourier-transform-based method using customwritten Mat lab codes. The final unwrapped phase images were averaged to improve the signal to noise ratio.

Results and discussion.-Spin configurations at surfaces and edges.-Figure 1(a) shows a schematic representation of edge twists and Skyrmions in a chiral nanostripe, whose width spans two edges and one Skyrmion. The colors denote in-plane $(x, y)$ magnetization components. This simplified model illustrates Bloch-type edge twists and a Skyrmion, in which the spins are perpendicular to radii that point outward from the center of the Skyrmion to its edge [see the magnified part of Fig. 1(a)]. Figure 1(b) shows a three-dimensional (3D) Skyrmion with chiral surface twists, which are characterized by an in-plane rotation angle $\theta$. Such twisted spin textures at the surface have been predicted to lower Skyrmion energetics and to provide thermodynamical stability of a Skyrmion lattice against the formation of conical states in a thin film $[9,10]$.

It should be noted that the formation of chiral surface and edge states results from the volume energy in the DMI term, without including modified magnetic parameters at the surface $[3,9,10]$. When considering magnetic properties that are specific to the surface, the edge spin configurations can be complex, and various forms of spin texture at

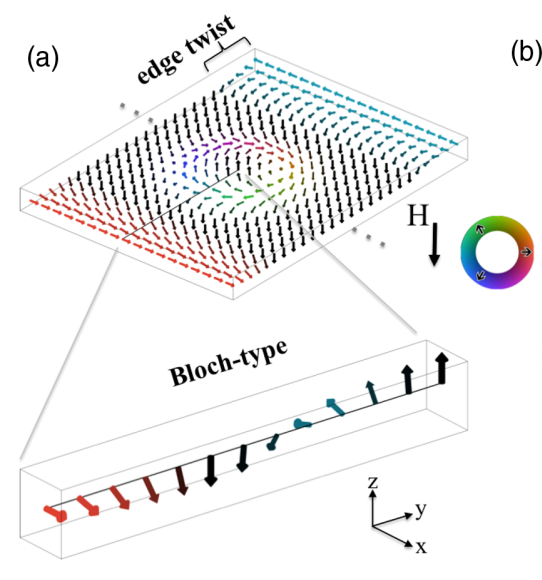

(b)

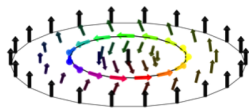

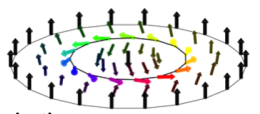
projection . -

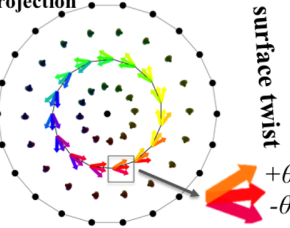

FIG. 1. Schematic representations of magnetic boundary states in a chiral magnet. (a) Spin texture of an edge Skyrmion in a chiral magnet nanostripe with chiral edge twists. In a Bloch-type Skyrmion, the spins are perpendicular to radii that point outwards from the Skyrmion center to its edge. (b) Three-dimensional Skyrmion model with chiral surface twists. The arrows represent the magnetization, while the directional color hue denotes the inplane $(x, y)$ magnetization components, according to the color wheel. The external magnetic field is applied along the $z$ axis.

specimen edges have been discussed theoretically in achiral and frustrated-type magnets [32].

Reconstruction of in-plane magnetization distributions from magnetic phase images.-We first used LM to visualize the magnetic-field-driven magnetization transitions in an FeGe nanostripe, as seen in the Supplemental Material [24] (Fig. S3). Figure 2(a) shows a representative LM image recorded at $95 \mathrm{~K}$ in a $300 \mathrm{mT}$ applied field, with the circular contrast features corresponding to individual magnetic Skyrmions. Strong intensity oscillations [a line profile in Fig. 2(b)], which arise from the abrupt sample thickness change and the defocused imaging conditions, complicate the interpretation of the weak magnetic contribution to the contrast at the edges. In Fig. 2(b), Skyrmion contrast features (marked "1" and "2") are much weaker than the oscillatory contrast (marked " $A$ " and " $B$ "). Retrieval of the magnetic structure at the specimen edges from such LM images is practically unfeasible [12].

To quantify the magnetic structure, we separated the magnetic contribution to the phase from the unwanted electrostatic (mean inner potential) contribution using off-axis EH, as described in Figs. 2(c)-2(e). Figure 2(c) shows the total (magnetic and electrostatic) phase $\varphi_{\mathrm{EM}}$ recorded from a region of the FeGe nanostripe at $95 \mathrm{~K}$ in an applied field of $300 \mathrm{mT}$. The electrostatic contribution to the phase $\varphi_{E}$ [Fig. 2(d)] was recorded at room temperature, above the Curie temperature $T_{C}(\sim 278 \mathrm{~K})$ for FeGe. Subtraction of the electrostatic phase $\varphi_{E}$ [Fig. 2(d)] from the total phase $\varphi_{\mathrm{EM}}$ [Fig. 2(c)] yields the magnetic phase $\varphi_{M}$ [Fig. 2(e)]. We then applied a model-based iterative reconstruction technique to such magnetic phase images to obtain local magnetization distributions. A short description 
(a)

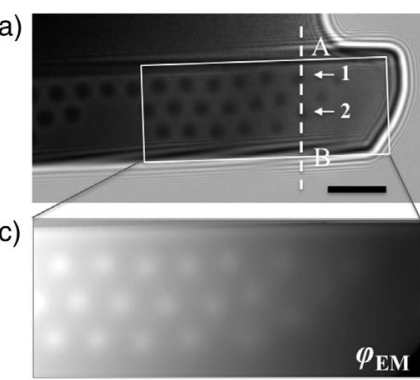

(d)
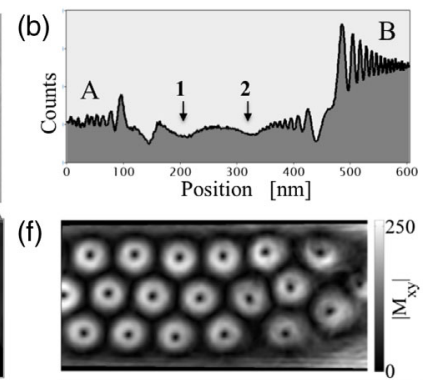

\section{(g)}

$\varphi_{\mathrm{E}}$

(e)

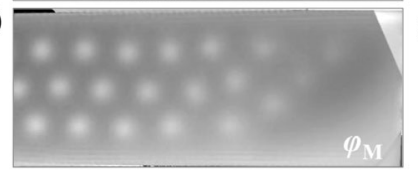

(a)

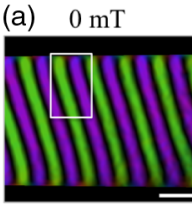

(e)
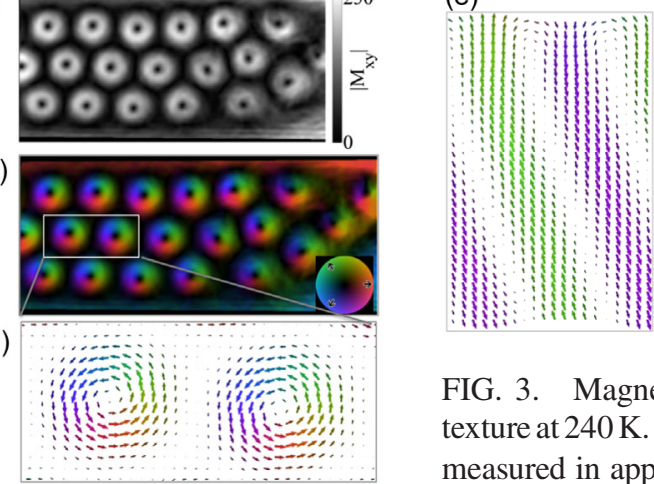

FIG. 2. Experimental retrieval of magnetic phase and reconstruction of in-plane magnetization. (a) Representative Lorentz TEM image taken at a defocus value of $-400 \mu \mathrm{m}$. The round contrast features in the $\mathrm{FeGe}$ nanostripe correspond to individual magnetic Skyrmions. The dashed line marks the position used to extract a line profile. (b) Line profile showing strong Fresnel fringe contrast oscillations at the specimen edges. (c) Total phase image $\varphi_{\mathrm{EM}}$ recorded using off-axis electron holography at $95 \mathrm{~K}$ in an applied field of $300 \mathrm{mT}$, including both electrostatic and magnetic contributions to the phase. (d) Electrostatic phase image $\varphi_{\mathrm{EM}}$ recorded at $295 \mathrm{~K}$ in an applied field of $300 \mathrm{mT}$. The Curie temperature of helimagnet $\mathrm{FeGe}$ is approximately $278 \mathrm{~K}$. (e) Magnetic phase image $\varphi_{M}$ obtained by subtracting $\varphi_{E}$ from $\varphi_{\mathrm{EM}}$. (f) Magnitude of in-plane magnetization $M_{x y}$ reconstructed from the magnetic phase using a model-based iterative reconstruction algorithm (see text for details). (g) Color representation of $M_{x y}$. The hue and brightness represent the direction and magnitude of $M_{x y}$, respectively, according to the color wheel. (h) Arrows representing $M_{x y}$ in the region marked in (g). The scale bar in (a) is $200 \mathrm{~nm}$.

of the approach and a workflow of the iterative algorithm are given in the Supplemental Material (Note 1 and Fig. S4). Figure 2(f) shows the magnitude $\left|M_{x y}\right|$ of the in-plane magnetization distribution reconstructed from Fig. 2(e). Figure $2(\mathrm{~g})$ shows the direction and magnitude of $M_{x y}$ in the form of color hue and brightness, respectively. Figure 2(h) shows an arrow plot of the reconstructed (in-plane) Skyrmonic spin texture from the region marked in Fig. 2(g).

Magnetic field dependence of edge twist and Skyrmion state.-Figures 3(a)-3(d) show in-plane magnetization $M_{x y}$ distributions in the FeGe nanostripe measured in different applied magnetic fields at 240 K. In Figs. 3(e)-3(h), arrows are used to represent the spins in the corresponding region marked in each $M_{x y}$ image [Figs. 3(a)-3(d)], in order to highlight the local spin texture of the chiral edge state.

When the FeGe nanostripe was zero-field cooled from room temperature to $240 \mathrm{~K}$, it was in a magnetic ground state of spin helices [Fig. 3(a)]. When a magnetic field of $100 \mathrm{mT}$ was applied normal to the plane of the nanostripe,
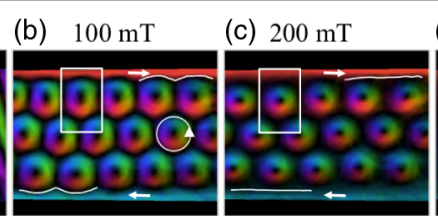

(d) $300 \mathrm{mT}$

(f)

(g)
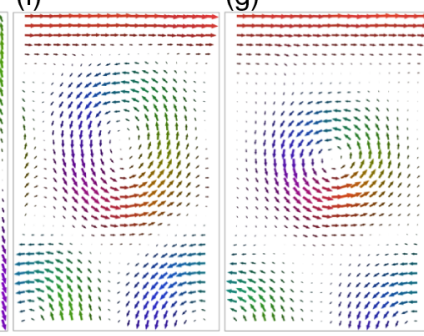

(h)

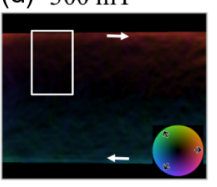

FIG. 3. Magnetic field dependence of the Skyrmion edge spin texture at $240 \mathrm{~K}$. (a)-(d) Reconstructed in-plane magnetization $M_{x y}$ measured in applied magnetic fields of 0, 100, 200, and $300 \mathrm{mT}$. The hue and brightness of the colors represents the direction and magnitude of $M_{x y}$, respectively, according to the color wheel in (d). The $M_{x y}$ maps were reconstructed from magnetic phase images using a model-based iterative reconstruction algorithm (see text for details). Lines are used to mark the boundaries between the edges and the Skyrmions. In order to highlight the chiral edge state, spins in the marked regions are shown in the form of arrows in (e)-(h). For ease of visualization, only $20 \%$ of the reconstructed spins are plotted. The scale bar in (a) is $100 \mathrm{~nm}$.

the spin helices evolved into a Skyrmion lattice [Fig. 3(b)]. The internal spin structures of individual Skyrmions adopted hexagonal rather than circular shapes, as a result of their proximity to each other in a lattice state $[18,21,33]$. Interestingly, the hexagonal Skyrmions are elongated in the vertical direction in the nanostripe. Quantitative measurements of the Skyrmion elongation are presented in Fig. 4(b). The elongation is thought to result from the geometrical confinement effect, with the Skyrmions flexibly changing their shape in response to the specimen geometry [19,20,22]. The elongated distortions became less pronounced with increasing applied magnetic field: the Skyrmions adopted more circular shapes and decreased in size in a $200 \mathrm{mT}$ field [Fig. 3(c)], and collapsed in a $300 \mathrm{mT}$ field [Fig. 3(d)].

The Skyrmions are always accompanied by chiral edge twists, as marked by white arrows in Figs. 3(b)-3(c) The chiral edge twists persist up to large values of applied magnetic field, when the Skyrmions collapse completely [Fig. 3(d)]. Such chiral edge states [see also the schematic diagram in Fig. 1(a)] are characterized by a twisted rotation of spins, and by nearly-in-plane spins at the very edge of the nanostripe [see red arrows at the edge in Figs. 3(f)-3(g)]. Close inspection of the edge twists and Skyrmions reveals that (i) the orientations of the edge twists at the upper and lower edges (white arrow pairs) are consistent with the spin rotation of the Skyrmionic textures [white circle arrow in Fig. 3(b)], (ii) the edge twists confine the Skyrmions and prevent them from stripping out of the nanostripe, with the Skyrmions moving towards the center of the nanostripe with 
increasing field, suggesting that the interaction between the edge and the Skyrmions is repulsive [8,34], (iii) the edge twists exhibit curved shapes at lower values of applied field [100 mT in Fig. 3(b)] and become less curved in higher fields [Fig. 3(c)], as denoted by white lines in Figs. 3(b), 3(c), and (iv) the edge states persist up to very high applied magnetic fields, at which the Skyrmions are transformed to a conical or a field-polarized ferromagnetic state [Fig. 3(h)] [22].

Characteristic dimensions of edge twist and confined Skyrmions.-We now quantify the characteristic length of the chiral edge twist and the sizes of confined Skyrmions in the $\mathrm{FeGe}$ nanostripe. Figure 4(a) shows reconstructed in-plane magnetization distributions under different temperature-field conditions, with dashed lines showing the positions of corresponding line profiles in Figs. 4(b)-4(c). The penetration length of the edge twist was measured between the nanostripe edge (marked "1") and the contrast dip (marked "2"), while the diameter of the Skyrmions was
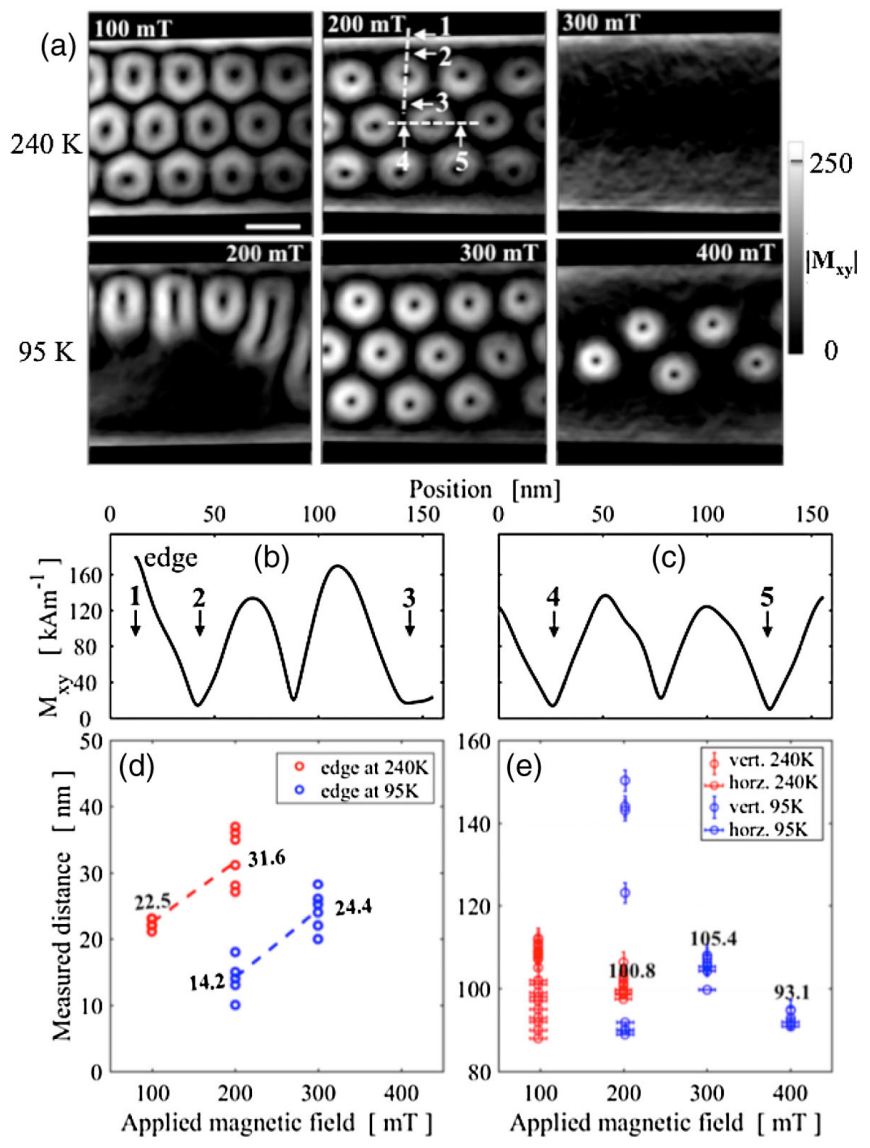

FIG. 4. Analysis of penetration lengths of chiral edge twists and sizes of Skyrmions in the confined FeGe nanostripe. (a) Reconstructed magnitude of in-plane magnetization $M_{x y}$ in the different field-temperature conditions indicated. (b),(c) Line profiles extracted from the regions marked by dashed lines in (a). (d) Penetration lengths of edge twists and (e) sizes of Skyrmions measured from (a). The average penetration lengths of the edge twists and the average diameters of the circular Skyrmions are indicated. The scale bar in (a) is $100 \mathrm{~nm}$. measured between the dips marked 2-3 and 4-5 in the line profiles. Figures 4(d)-4(e) summarize the measurements, which reveal that the edge twist expands slightly towards the nanostripe interior with increasing applied field [see the dashed lines at 95 and $240 \mathrm{~K}$ in Fig. 4(d)]. The measured values $(14.2,24.4,22.5$, and $31.6 \mathrm{~nm})$ are a fraction of the helix period $L_{D}=70 \mathrm{~nm}$ for FeGe [35], and are in good agreement with predicted penetration lengths $[7,8,11]$. For the confined Skyrmions, (i) in lower applied magnetic fields, the Skyrmions exhibit a strong elongation [see the horizontal and vertical measurements in Fig. 4(e)], (ii) in higher applied magnetic fields, the Skyrmions become round, with diameters that are larger than the $80 \mathrm{~nm}$ characteristic diameter of Skyrmions in an extended thin film [35]. The formation of elliptically deformed Skyrmions with large diameters can be ascribed to a strong confinement effect, as discussed recently for a single Skyrmion chain in an FeGe nanostripe [22]. In the present study, the width of the nanostripe $(\sim 330 \mathrm{~nm})$ spans a pair of edge twists and three rows of Skyrmions. In a nanostripe whose width is larger or smaller, Skyrmions are anticipated to flexibly change their sizes and shapes to accommodate the sample geometry, in accordance with the topological invariance of Skyrmionic spin textures in confined nanostructures.

Quantitative analysis of saturation magnetization.-We further analyzed the reconstructured magnetization distributions to determine the saturation magnetization in the FeGe nanostripe. According to analytical two-dimensional spin models for a magnetic helix and a Skyrmion $[17,36]$, the saturation magnetization $M_{S}$ can be extracted from the maximal in-plane magnetization. We therefore measured $M_{S}$ in selected regions (dashed lines) for the helix and Skyrmion in Figs. 5(a), 5(b) respectively, in order to determine the maximal in-plane magnetization. The procedure is described in Fig. S5 in the Supplemental Material [24]. To minimize measurement errors, measurements were performed on individual helices and Skyrmions to provide histograms of $M_{S}$ at 95 and $240 \mathrm{~K}$ [Figs. 5(c)-5(f)]. The histograms were then fitted with normal distribution functions, to yield mean values of $M_{S}$, with their full widths at half maximum used to define measurement errors. The measured values of $M_{S}$ at $240 \mathrm{~K}(136 \pm 27$ and $125 \pm$ $33 \mathrm{kA} \mathrm{m}^{-1}$ for the helix and Skyrmion, respectively) are approximately half of those measured at $95 \mathrm{~K}(256 \pm 42$ and $239 \pm 41 \mathrm{kA} \mathrm{m}^{-1}$ for the helix and Skyrmion, respectively), as a result of thermal magnetization reduction. The resulting temperature-dependent magnetization curve, which takes the form $M_{S}=M_{0}\left(1-T / T_{C}\right)^{\beta}$, is shown in Fig. 5(g), where the saturation magnetization in the zero temperature limit [37] $M_{0}=376 \mathrm{kA} \mathrm{m}^{-1}$, the critical exponent [37] $\beta=0.336$, and the Curie temperature [37] $T_{C}=278 \mathrm{~K}$. The experimentally measured $M_{S}$ values [squares and diamonds in Fig. 5(g)] are compared with the expected values from the magnetization curve [solid circles in Fig. 5(g)]. The measured values of $M_{S}$ are smaller than 

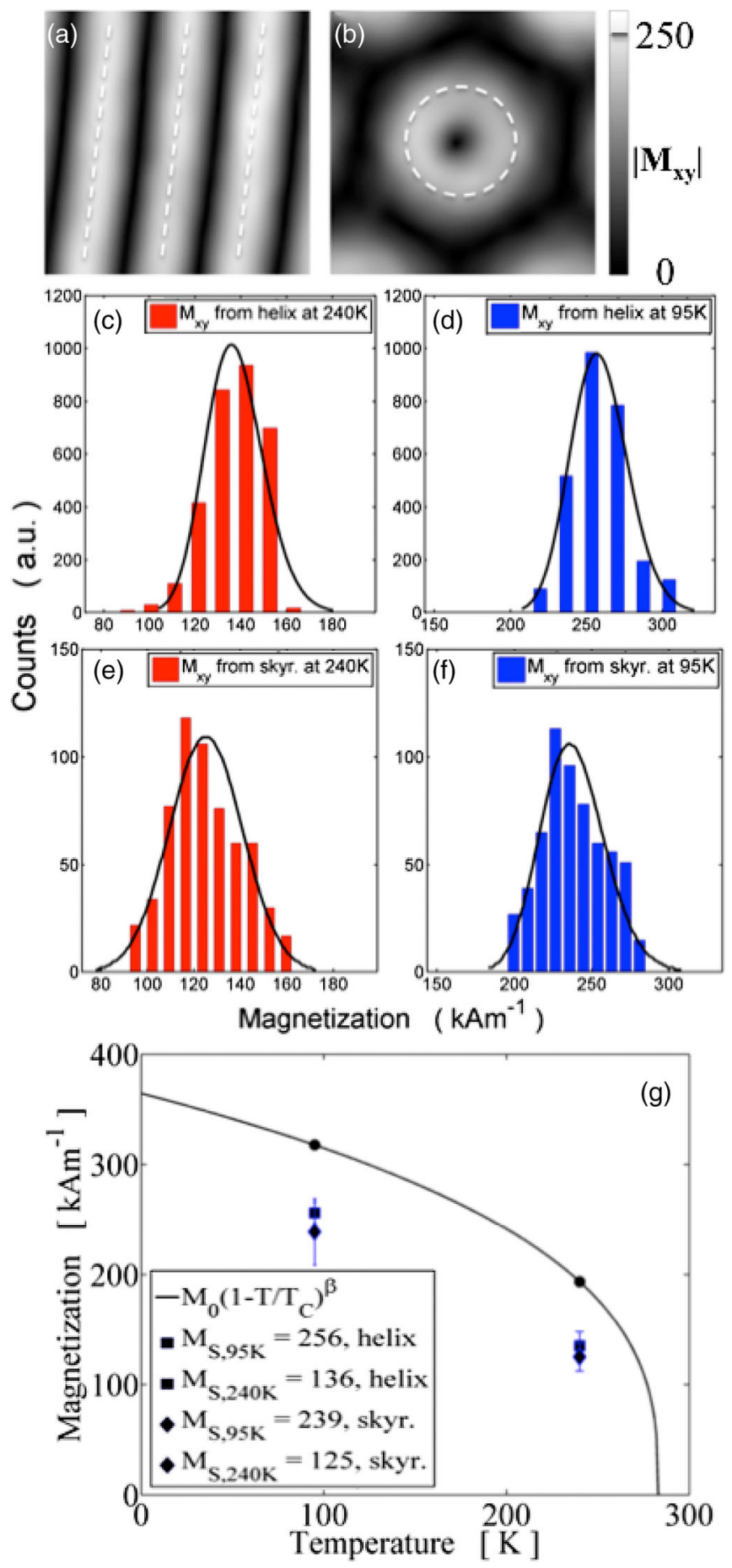

FIG. 5. Analysis of projected in-plane magnetization. (a),(b) Magnitude $\left|M_{x y}\right|$ of in-plane magnetization for helices and Skyrmions measured at $95 \mathrm{~K}$ in an applied magnetic field of $300 \mathrm{mT}$. The dashed lines mark positions used for magnetization determination. (c)-(f) show corresponding histograms and fits to normal distribution functions. (g) Measured saturation magnetization for helices and Skyrmions at 95 and $240 \mathrm{~K}$, plotted alongside a measured magnetization curve for a critical exponent $\beta$ of 0.336 (see text for details). the expected values. For example, $M_{S, 95 K}=256 \mathrm{kA} \mathrm{m}^{-1}$ measured from the helices is approximately $80 \%$ of the expected value of $318 \mathrm{kA} \mathrm{m}^{-1}$. This discrepancy is likely to result from a nonmagnetic surface layer on the TEM specimen, as well as from possible inaccuracy in the determination of the specimen thickness [33].

Significantly, $M_{S}$ at $95 \mathrm{~K}(240 \mathrm{~K})$ measured for the Skyrmions is $\sim 7 \%(\sim 9 \%)$ lower than that measured from the helices. This difference can be ascribed to the 3D nature of the Skyrmion model [Fig. 5(b)], which contains chiral surface twists [9]. A decrease in magnetic phase shift recorded using off-axis EH is therefore expected, resulting in an underestimate of the saturation magnetization $M_{S}$ from a measurement of the maximum in-plane magnetization of Skyrmions. Our comparative analysis of $M_{S}$ for helices and Skyrmions supports a theoretically predicted 3D Skyrmion model with chiral surface twists [9].

Conclusions. - In summary, we have investigated chiral boundary (surface and edge) states in an FeGe nanostripe using off-axis electron holography, with a focus on the quantitative understanding of the in-field evolutions and characteristic penetration lengths of edge twists. The analysis of magnetization distributions reconstructed from electron holographic phase images for edge twists and Skyrmions allows precise measurements of the characteristic sizes of edge states and confined Skyrmions in the FeGe nanostripe. We quantified the saturation magnetization at 95 and $240 \mathrm{~K}$ from the maximal values of the reconstructed inplane magnetization for the helices and Skyrmions. An observed difference in measured saturation magnetization between helices and Skyrmions was attributed to the 3D nature of the Skyrmion model, which involved chiral surface twists. Such magnetic boundary states are important for the exploitation of Skyrmion-based devices, as they affect the particlelike properties of topologically protected spin textures and are likely to have a strong effect on magnetotransport properties $[8,34,38]$ and spin wave excitations [39] in chiral magnet nanostructures. From a methodological perspective, the experimental approach that we use opens new avenues for exploring quantum confinement in other complex noncollinear spin systems at the nanoscale.

We thank Nikolai S. Kiselev and Filipp N. Rybakov for helpful discussions. This work was supported financially by the Chinese National Natural Science Foundation (11774403, 11374174, 51390471, 51527803, 11474290) and the National Key Research and Development Program of China (2016YFB0700402, 2017YFA0303000), and National 973 Project of China (2015CB654902). D. S. acknowledges a Scholarship for Overseas Graduate Studies at Tsinghua University. Z.-A. L. is grateful to the Hundred Talent Program B of the Chinese Academy of Sciences. The research leading to these results has received funding from the European Research Council under the European Union's Seventh Framework Programme (FP7/20072013)/ ERC Grant Agreement No. 320832. 
*zali79@iphy.ac.cn

[1] X. G. Wen, Int. J. Mod. Phys. B 06, 1711 (1992).

[2] M. Hasan and C. L. Kane, Rev. Mod. Phys. 82, 3045 (2010).

[3] S. Rohart and A. Thiaville, Phys. Rev. B 88, 184422 (2013).

[4] N. Nagaosa and Y. Tokura, Nat. Nanotechnol. 8, 899 (2013).

[5] I. Dzyaloshinskii, J. Phys. Chem. Solids 4, 241 (1958).

[6] T. Moriya, Phys. Rev. 120, 91 (1960).

[7] S. A. Meynell, M. N. Wilson, H. Fritzsche, A. N. Bogdanov, and T. L. Monchesky, Phys. Rev. B 90, 014406 (2014).

[8] J. Sampaio, V. Cros, S. Rohart, A. Thiaville, and A. Fert, Nat. Nanotechnol. 8, 839 (2013).

[9] F. N. Rybakov, A. B. Borisov, and A. N. Bogdanov, Phys. Rev. B 87, 094424 (2013).

[10] A. O. Leonov, Y. Togawa, T. L. Monchesky, A. N. Bogdanov, J. Kishine, Y. Kousaka, M. Miyagawa, T. Koyama, J. Akimitsu, T. Koyama et al., Phys. Rev. Lett. 117, 087202 (2016).

[11] M. N. Wilson, E. A. Karhu, D. P. Lake, A. S. Quigley, S. Meynell, A. N. Bogdanov, H. Fritzsche, U. K. Rossler, and T. L. Monchesky, Phys. Rev. B 88, 214420 (2013).

[12] H. Du, R. Che, L. Kong, X. Zhao, C. Jin, C. Wang, J. Yang, W. Ning, R. Li, X. Chen et al., Nat. Commun. 6, 8504 (2015).

[13] S. Heinze, K. von Bergmann, M. Menzel, J. Brede, A. Kubetzka, R. Wiesendanger, G. Bihlmayer, and S. Blügel, Nat. Phys. 7, 713 (2011).

[14] S. H. Woo, K. Litzius, B. Krueger, M. Y. Im, L. Caretta, K. Richter, M. Mann, A. Krone, R. M. Reeve, M. Weigand et al., Nat. Mater. 15, 501 (2016).

[15] P. Milde, D. Koehler, J. Seidel, L. M. Eng, A. Bauer, A. Chacon, J. Kindervater, S. Muehller, C. Pfleiderer, S. Buhrandt et al., Science 340, 1076 (2013).

[16] X. Z. Yu, Y. Onose, N. Kanazawa, J. H. Park, J. H. Han, Y. Matsui, N. Nagaosa, and Y. Tokura, Nature (London) 465, 901 (2010).

[17] H. S. Park, X. Yu, S. Aizawa, T. Tanigaki, T. Akashi, Y. Takahashi, T. Matsuda, N. Kanazawa, Y. Onose, D. Shindo et al., Nat. Nanotechnol. 9, 337 (2014).

[18] K. Shibata, A. Kovács, N. S. Kiselev, N. Kanazawa, R. E. Dunin-Borkowski, and Y. Tokura, Phys. Rev. Lett. 118, 087202 (2017).

[19] Z.-A. Li, F. Zheng, A. H. Tavabi, J. Caron, C. Jin, H. Du, A. Kovács, M. Tian, M. Farle, and R. E. Dunin-Borkowski, Nano Lett. 17, 1395 (2017).

[20] T. Matsumoto, Y.-G. So, Y. Kohno, H. Sawada, Y. Ikuhara, and N. Shibata, Sci. Adv. 2, e1501280 (2016).

[21] D. McGrouther, R. J. Lamb, M. Krajnak, S. McFadzean, S. Mcvitie, R. L. Stamps, and A. O. Leonov, New J. Phys. 18, 095004 (2016).
[22] C. Jin, Z.-A. Li, A. Kovács, J. Caron, F. Zheng, F. N. Rybakov, N. S. Kiselev, H. Du, S. Blügel, M. Tian et al., Nat. Commun. 8, 15569 (2017).

[23] F. Zheng, H. Li, S. Wang, D. Song, C. Jin, W. Wei, A. Kovács, J. Zang, M. Tian, H. Du et al., Phys. Rev. Lett. 119, 197205 (2017).

[24] See Supplemental Material at http://link.aps.org/ supplemental/10.1103/PhysRevLett.120.167204 for detailed descriptions of structural characterization and thickness measurement of the FeGe nanostripe, Lorentz Fresnel images, a workflow for the model-based iterative magnetization reconstruction algorithm, and histograms of maximal in-plane magnetization measured from individual helices and Skyrmions, which includes Refs. [25-31].

[25] J. Caron, Ph.D. thesis, Aachen University (RWTH), 2017.

[26] M. Mansuripur, J. Appl. Phys. 69, 2455 (1991).

[27] R. E. Dunin-Borkowski et al., Microsc. Res. Technol. 64, 390 (2004).

[28] H. W. Engl, M. Hanke, and A. Neubauer, Regularization of Inverse Problems (Kluwer Academic, Dordrecht and Boston, 1996).

[29] A. N. Tikhonov and V. I. Arsenin, Solutions of Ill-Posed Problems, Scripta Series in Mathematics (Winston and Distributed solely by Halsted Press, Washington and New York, 1977).

[30] K. M. Krishnan, Fundamentals and Applications of Magnetic Materials, 1st ed. (Oxford University Press, Oxford, 2016).

[31] K. Iakoubovskii et al., Microsc. Res. Technol. 71, 626 (2008).

[32] A. O. Leonov and M. Mostovoy, Nat. Commun. 8, 14394 (2017).

[33] A. Kovács, J. Caron, A. S. Savchenko, N. S. Kiselev, K. Shibata, Z.-A. Li, N. Kanazawa, Y. Tokura, S. Blügel, and R. E. Dunin-Borkowski, Appl. Phys. Lett. 111, 192410 (2017).

[34] X. Zhang, G. P. Zhao, H. Fangohr, J. P. Liu, W. X. Xia, J. Xia, and F. J. Morvan, Sci. Rep. 5, 7643 (2015).

[35] X. Z. Yu, N. Kanazawa, Y. Onose, K. Kimoto, W. Z. Zhang, S. Ishiwata, Y. Matsui, and Y. Tokura, Nat. Mater. 10, 106 (2011).

[36] A. N. Bogdanov and A. Hubert, J. Magn. Magn. Mater. 138, 255 (1994).

[37] L. Zhang, H. Han, M. Ge, H. Du, C. Jin, W. Wei, J. Fan, C. Zhang, P. Li, and Y. Zhang, Sci. Rep. 6, 22397 (2016).

[38] J. Müller, New J. Phys. 19, 025002 (2017).

[39] T. Schwarze, J. Waizner, M. Garst, A. Bauer, I. Stasinopoulos, H. Berger, C. Pfleiderer, and D. Grundler, Nat. Mater. 14, 478 (2015). 